\title{
Digital Support for Living with and Beyond Gynaecological Cancer
}

\section{Structured abstract}

\section{Introduction}

Gynae-Radiotherapy places exceptional psychosocial and physical burdens on patients. Technological developments and associated acute toxicity and survival outcomes have improved, however holistic support has not kept pace. Digital technologies have potential to enhance support and patient experience.

The project aimed to co-create a prototype of a digital health intervention that could serve the needs of women living with and beyond treatment for gynaecological cancer.

\section{Methods}

A multi-disciplinary and co-creation approach was adopted. Four workshops were held, comprising of a number of activities to support participants' expression of views and facilitate discussion. Methods included word cloud generation, prompt cards, empathy maps and persona creation, domain storylines and requirements identification.

Results

Support drops off dramatically once treatment is completed. Patients struggled to adjust to their 'new normal' and felt unprepared for changes post-treatment. Patients felt overwhelmed with leaflets yet wanted instant access to reliable and relevant information in one place, better information on late side effects and improved communication about sexual health and sexuality. Reassurance through a digital intervention was viewed positively and specific ideas for achieving this were suggested through: Sharing experiences; targeted practical advice; peer support and advice/support for significant others.

\section{Conclusion}

The co-creation of a prototype generated further discussion and an interactive prototype was developed. Based on workshop findings it is believed that the intervention could provide life-long support for women living with and beyond cancer.

\section{Implications for practice}

Increased focus is needed on the late effects of radiotherapy, specifically in supporting psychosocial wellbeing. Co-creation is a rewarding and fulfilling activity that met numerous aims beyond those of the project. It is recommended that mixed staff-patient groups are developed and adopted in more informal ways for the improvement of services.

Keywords

Gynaecological Cancer; Patient Support; Radiotherapy; Co-design; Digital Intervention; Patient Wellbeing. 


\section{Main Article}

\section{ntroduction}

Radiotherapy treatment for pelvic cancers places exceptional burdens on patients (1). Specifically, radiotherapy for gynaecological cancers can cause erythema and desquamation on the genitals and pelvic region for more than six months following treatment and other side effects of radiotherapy can occur months or years after treatment finishes. Reported quality of life significantly worsens as patients experience acute side effects, improving once these transient effects begin to improve (2). However, chronic side effects include psychosocial and physical symptoms including depression, anxiety, fear of dying, fatigue, pain, bladder dysfunction and irritation, proctitis, impaired bowel health, vaginal stenosis, weakening of the vaginal walls with associated prolapse, infertility, and premature menopause (3-6). Cancer-related fatigue affects over half women treated with a higher proportion in cervical cancer interfering with an individual's ability to carry out daily activities (7). Moreover, treatment procedures have significant effects on health, body image, gender role functioning (femininity), sexual functioning and fertility (8-10).

Advances in technological treatment techniques are being made, notably IMRT and VMAT that allow precise conformity to the PTV while sparing surrounding OAR. Similarly, Image Guided Adapted Brachytherapy using MRI, has been shown to improve local control rates (11). Developments in treatment have improved outcomes in both survival and acute toxicity, however the physiological and psychological impact of diagnosis and treatment should not be underestimated.

Equal attention and effort are required to develop holistic support, as often support systems have not kept pace with technological developments. Specifically, follow-up support in primary and secondary care has not focused on monitoring symptoms or psychological side effects (12). 30\% of cancer patients experience moderate or severe unmet supportive care needs after treatment, most of which could be met by AHPs (13). However, generally patients have expressed concern that follow-up oncology appointments prioritise 'survival' at the expense of experiential issues of living with and beyond cancer and that healthcare teams lack the time and expertise to handle unmet needs of patients (14).

Being able to express sexual interests and preferences is crucial to mental health and for many women is compromised through the process of being a gynaecological cancer patient (15). Many women experience dissatisfaction with their sexual activity post-treatment and, according to Bakker et al (2017), there are no published effective sexual rehabilitative interventions to support sexual recovery of gynaecological cancer patients (16). Barriers to providing psychosexual support include organizational issues of information and care, staff skills, knowledge, and training (17).

\section{Supporting Patients Digitally}

Digital technologies are central to a number of changes in the NHS long-term plan (18). The 2015 report from the British Independent Cancer Taskforce highlights the potential of digital technologies to revolutionize patient information and improve patient experience and recovery (19). For people living with a health condition, several motivations surrounding participation in online health communities are reported $(20,21)$. For example, to seek social support from others who have similar experiences and can thus offer empathy and compassion in ways others cannot (21) and to offer condition specific, stigma-free spaces 
comprised of people representing a diversity of experiences and advice (22). Access to these online resources has been found to correlate with better medical knowledge, which can facilitate patient-clinician interactions and increase confidence around decision-making (20).

However, the NHS has a poor history of technology adoption (23) and digital technologies in care are controversial, heralded as facilitating patient empowerment and improving care, yet also potentially promoting isolation and distributing caring responsibilities in problematic ways. While the NHS are commissioning services to develop trusted app repositories, they have not been integrated into practices and care pathways to support patients to live well with and beyond cancer. Consequently, the impact of using digital platforms by patients is unknown. Despite highly cited promise, how these systems impact on daily lives and in what ways they change patient experiences is unclear. Yet, a simple digital support intervention could provide additional support to patients through and beyond their treatment.

This project used the phrase "digital health intervention," to enable flexibility of interpretation for participants. Digital Health Intervention represents digital technology that is used to achieve health sector objectives (24).

\section{Co-Creation}

Working closely with people in the design of services - particularly within the sensitive domain of healthcare-is necessary to gain dynamic understandings of the person's lived experiences, needs and values. Without such insight, assumptions can be made that overlook how users experience and value interventions, obscuring the reasons and requirements for user engagement.

Within cancer services, there has been increasing awareness of the importance of involving end users in design of practices. The Society and College of Radiographers response to engagement in the NHS Five Year Forward View stated that previous patient and public partnerships have often been implicit rather than explicit, advocating for a clear direction towards a shared overall vision $(25,26)$.

Users can be involved in design processes in a variety of ways, from consultation about needs, as participants in usability testing, as well as deeper participatory involvement throughout the entire process (27). Co-creation, or participatory design, focuses on bottomup, grassroots involvement throughout design processes, from idea formulation through to trialling and evaluation.

In the context of self-monitoring and management, user involvement is vital as individual motivation for use is a critical factor for initial and continued use. Poorly designed, difficult to use, or difficult to integrate interventions can deter long-term engagement.

\section{Aim}

The aim of the project was to co-create a prototype digital health intervention to serve needs of women living with and beyond treatment for gynaecological cancer. 


\section{Methods}

To meet the project aim, a multi-disciplinary and co-creation approach, informed by the NIHR standards for public involvement, was adopted (28). Four workshops were held (Table 1).

Table 1: Intended and actual participants for workshops

\begin{tabular}{|c|c|c|c|}
\hline $\begin{array}{l}\text { Workshop } \\
\text { Number }\end{array}$ & Participants (Intended) & Participants (Actual) & $\begin{array}{l}\text { Objectives for } \\
\text { Session }\end{array}$ \\
\hline 1 & $\begin{array}{l}\text { Staff Members x } 5 \\
\text { Researchers x } 3\end{array}$ & $\begin{array}{l}\text { Staff members x } 4 \text { (s1, s2, } \\
\text { s3, s4) } \\
\text { Researchers x } 3(\mathrm{LW}, \mathrm{HS} \text {, } \\
\text { DH) } \\
\text { Designer } \times 1(\mathrm{KE})\end{array}$ & $\begin{array}{l}\text { Establish deep } \\
\text { understanding of } \\
\text { support available } \\
\text { and the treatment } \\
\text { pathway }\end{array}$ \\
\hline 2 & $\begin{array}{l}\text { Patients } \times 5 \\
\text { Researchers } \times 2\end{array}$ & $\begin{array}{l}\text { Patients x } 1 \text { ( } 1 \text { 1) } \\
\text { Researchers } \times 2(\mathrm{LW}, \mathrm{HS})\end{array}$ & $\begin{array}{l}\text { Establish key areas } \\
\text { for supporting } \\
\text { women following } \\
\text { treatment for } \\
\text { gynaecological } \\
\text { cancer. }\end{array}$ \\
\hline 3 & $\begin{array}{l}\text { Staff } \times 3 \\
\text { Patients } \times 3 \\
\text { Researchers } \times 2\end{array}$ & $\begin{array}{l}\text { Staff } \times 2(s 1, \mathrm{~s} 5) \\
\text { Patients } \times 4(\mathrm{p} 2, \mathrm{p} 3, \mathrm{p} 4, \\
\text { p5) } \\
\text { Researchers } \times 2 \text { (LW, HS) }\end{array}$ & $\begin{array}{l}\text { Development of } \\
\text { design brief and } \\
\text { requirements for } \\
\text { the system }\end{array}$ \\
\hline 4 & $\begin{array}{l}\text { Staff } \times 3 \\
\text { Patients } \times 3 \\
\text { Researchers } \times 3 \\
\text { Designer } \times 1\end{array}$ & $\begin{array}{l}\text { Staff } \times 2(\mathrm{~s} 2, \mathrm{~s} 3) \\
\text { Patients } \times 2(\mathrm{p} 2, \mathrm{p} 3) \\
\text { Researchers } \times 3(\mathrm{LW}, \mathrm{HS}, \\
\text { DH) } \\
\text { Designer } \times 1 \text { (KE) }\end{array}$ & $\begin{array}{l}\text { Review of initial } \\
\text { wireframes }\end{array}$ \\
\hline
\end{tabular}

Staff members included Therapeutic Radiographers, Macmillan Support, On Treatment Review Radiographers, Specialist Trainees in Oncology and Gynae Cancer Nurse Specialist. A total of five staff members attended at least one workshop.

Eligibility criteria for patients were:

- Previous diagnosis of gynaecological cancer

- Received radiotherapy treatment for gynaecological cancer (including brachytherapy, adjuvant and neo-adjuvant chemotherapy) that completed more than six months from the date of the first workshop 
- Capacity to independently consent to participation

- Understanding of verbal and written material in English.

Following the first two workshops, key elements for attention were established. Because only one patient attended the second workshop, the format of the workshop was adjusted to enable a safe and informal environment for discussion and more key areas for support were elicited in workshop three.

The key elements fed into workshop three, following which an intervention design brief or 'wish list' was created and shared with the digital design company. Initial wireframes, a visual guide that represented the skeletal framework of the intervention was then developed.

The final workshop involved staff and patients who had all previously attended a session. The wireframes were presented to elicit discussion and additional system requirements. These requirements were fed into the next stage of design, development of interactive wireframes.

During the workshops, activities and resources were used to support participants to express views and stimulate discussions. A workshop guide is in Appendix A. Workshops were voice recorded and summaries were written from the recordings.

Methods included:

Wordclouds using web hosted application www.mentimeter.com. Using mobile phones, participants submitted words or phrases in response to the questions: What words come to mind when you hear the phrase, "digital intervention"? and in one word, describe the current levels of support for women following treatment for gynaecological cancer? Resulting wordclouds were then used to generate discussion.

Prompt Cards, with factors affecting wellbeing, were laid out on the table (see Appendix A). Participants were asked to select one that resonated with their experiences following treatment for gynaecological cancer, stimulating discussion.

Empathy Maps and Persona Creation encouraged participants to think about emotions associated with using health interventions. The Personas technique generated understanding of system users' characteristics, needs and goals (29). Empathy maps enabled an exploration of patients' post-treatment experiences and associated thoughts and feelings (see Appendix B for example).

Domain Storylines generated from a starting point of diagnosis, participants discussed actors involved, events pivoting off this main event, commands, instructions, systems, policies and procedures, things that worked and things that broke down. Areas of focus were potential threats to wellbeing, support available, communication, pathways, and gaps in provision.

The storyline was drawn on white board paper and allowed participants to stand up and contribute.

Requirements Identification is the process of determining user expectations for a new or modified product. Requirements must be quantifiable, relevant and detailed and analysis takes place in iterative cycles to identify and distinguish essential user requirements building the development path. Participants completed requirements templates during and 
after workshops. Facilitators also completed requirements templates to ensure discussions were being fed into the design process.

Recruitment of Participants

\section{Individuals who have received Radiotherapy for Gynaecological Cancer}

Initially, participants were to be recruited from the local Cancer Alliance Partnership Group. However, once recruitment started, it became clear that the number of eligible participants in the group was not sufficient. The alternative recruitment method was adopted, recruiting patients from follow-up clinics, enabling more patients to participate in workshops.

Recruitment methods are shown in Figure 1.

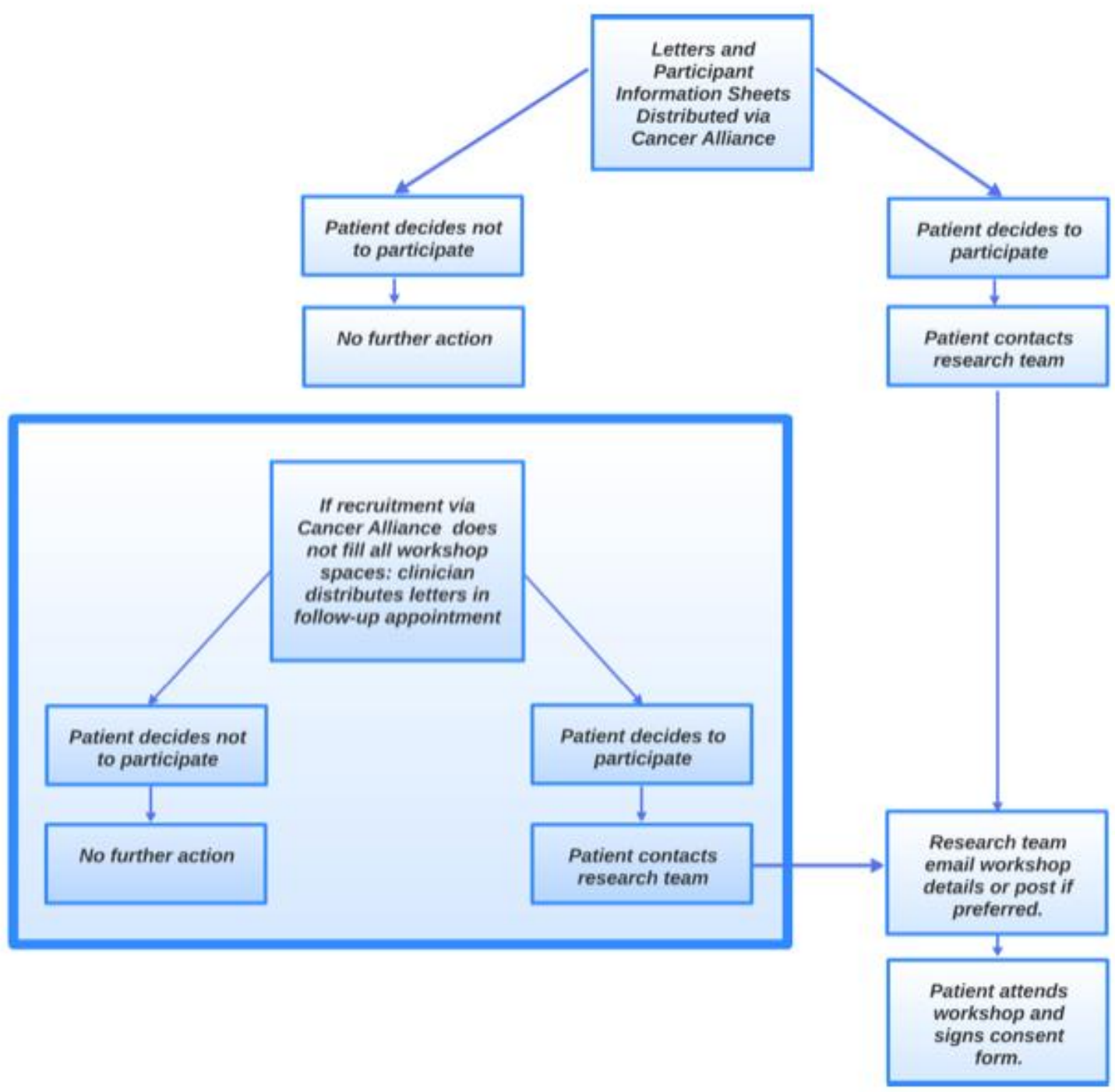

Figure 1: Flow diagram showing patient recruitment procedure

Participants could attend any number of workshops. Each workshop started with a project overview to support any new participants and also an update on the previous stages. 


\section{Staff Members}

A purposive approach to staff recruitment was adopted to secure multi-professional representation and ensure the voices of key stakeholder groups were included in the cocreation process.

The project was approved by the NHS Research Ethics Committee (IRAS project ID: 262882) and by the Health Research Authority. Written consent was obtained from all participants at the start of each workshop. Travel reimbursement was offered to all participants and a $£ 20$ high street voucher was given as a token of thanks for participation after each workshop.

Results

Current issues with support

Workshop discussions identified that support drops off dramatically after treatment and that patients felt unprepared for changes to wellbeing post-treatment. Although a 6-week post-treatment triage service exists, this service focuses on supporting patients experiencing acute symptoms of radiotherapy treatment. Staff raised broader questions regarding responsibility for supporting patients once this window of treatment/support ends and that signposting to support/resources after treatment is lacking. Staff discussed Holistic Needs Assessments and cancer rehabilitation/support services and resources currently available within the trust which could address unmet needs. However, staff were unconvinced these services were being utilised effectively and whether they were meeting patients' needs in a meaningful way.

Participants expressed that follow-up appointments are time constrained and restricted to a focus on physical symptoms and scans which precludes other aspects of wellbeing. As one patient encapsulated "all they [consultants] ask is 'has there been any bleeding?' No, and that's it!"

Support for patients post-treatment was unanimously seen as lacking and contributed to feelings of abandonment and isolation:

“Once you've had your treatment, you ring the bell, and you go, and then you just go,

'well where do I go now, what do I do?' You've had this team of people all fighting

with you, sort of looking after you, and then say, 'off you go.' Well, what do I do?

Where do I go? That's scary as well". (P1)

Although leaflets containing information related to treatment/recovery are provided, participants expressed that the volume and presentation of information is overwhelming and not always helpful, leaving patients to "muddle through" their new normal with little guidance. That patients' suffering is taking place under the radar and not being properly addressed was felt particularly strongly within the staff workshop:

"I have spoken to loads of patients who have had long-term problems relating to radiotherapy, maybe 15,20 years ago, who have said to me, but my cancer is cured, so actually these are things that I will put up with. And the people who come in are the 
people who are struggling... and they're the ones that shout the loudest, and you worry about all the others who are just quietly putting up with it." (S2)

This issue of hidden/necessary suffering recurred in other workshops where patients discussed chronic side effects and lifestyle adaptations as the price to be paid for survival or minimised their experiences in comparison with others' suffering:

P2: “...Diet is very important, I do have... damage to the bowel...and I do get nervous, and if you're worried about something, that's it, straight to the loo two or three times a day, I mean, it's not the runs... "

LW: "And do you feel comfortable with those diet changes and the not drinking alcohol and no takeaways?"

P2: “Yeah I do, yeah. I'm just glad to be alive...I mean, I don't know if you watch DIY SOS, some of the poor people on that, what a life they have, stuck in a wheelchair"

P3: "yeah, there's always someone who has it worse".

She later admitted that changes to her bowel function and diet had been embarrassing, affected her mood and prevented her from staying overnight at family members' homes, a problem she dealt with for six years: "But that's just part of what can happen to you, y'know?".

Long-term problems with bowel/bladder urgency were reported by other patients, as well as pain and discomfort during sex:

"it's red raw at first, the pain, it goes after a bit, but l've got to get past that bit, it's horrible, it's always the same, red raw... I just wish I could put something on to numb it, it's red raw, inside, not the whole time because then I just couldn't do it, l've just got to get past that bit." (P3)

This prompted a staff participant to suggest a number of lubricants which might help, something the patient was unaware of. Patients felt that information on sexual health/ sexuality was lacking and not well handled, with a consensus on the need to improve education and communication on the use of dilators post-treatment. As one patient remarked "I got told if I didn't use it, it would close up - it didn't! [laughs]". Additionally, staff were concerned about the lack of training and information to support LGBTQI patients.

Despite numerous challenges to physical and psychosocial wellbeing, all patients discussed navigating their new normal as a case of "keeping busy" and "getting on with it". All of the women spoke of being told they "looked well" even though the reality was sometimes quite different. Fear of recurrence and health anxiety was common but was something the women kept to themselves, not wishing to "burden" others or discuss "embarrassing" issues. Hidden suffering was therefore compounded by the often invisible nature of late side effects and associated taboos. 


\section{Plugging the gap}

In the absence of formally integrated post-treatment support, participants drew on a range of resources and services provided by charitable support organisations. Services included:

- Peer support groups

- 1-2-1 psychological therapy

- Mindfulness courses

- Holistic therapies (raiki, massage, meditation, beauty therapy)

- Lifestyle classes (diet, exercise)

Participants also used digital interventions such as Headspace and apps which provided relaxing music/sounds. Participants did not seek support from GPs/clinical staff. This was due to a lack of time/confidence to make appointments but it was also felt by some that "I just don't think the doctors get it" (P1).

Although services provided by charitable organisations were celebrated, participants discussed barriers to accessing these resources such as time and travel. Concerns were raised regarding what a person could access when experiencing periods of extreme low mood and anxiety, especially outside of 'office hours'. Additionally, participants were unsure about accessing services designed for cancer patients as they considered themselves to be "out of the other side".

As such, having reassurance through a digital intervention was viewed positively, providing people with reliable and relevant information in one place, which could be accessed at any time. Discussions reiterated the need for support beyond the current pathway addressing more holistic and long-term aspects of wellbeing. Some unmet needs that the intervention could target included:

- $\quad$ support for mental/psychosexual wellbeing

- $\quad$ support returning to work

- $\quad$ adjusting to the 'new normal'

- late side effects advice

- Financial advice (incl. welfare/benefits)

- lifestyle advice (diet, recipes, exercise)

- support for family/friends

- facilitating peer support.

Although participants raised the issue of being tech savvy enough to use an app, staff suggested that the intervention could be incorporated early within pathways and that staff could support patients in using it.

\section{Wish List Ideas}

Patients envisaged a resource that provided information, which did not overwhelm/frighten them, which they could turn to when anxious. Although patients imagined an intervention primarily for patients, staff emphasised the need for support/information for significant others and advice on dismantling taboos/having difficult conversations. It was also suggested that the intervention could provide information on advances in patient care posttreatment (e.g. new lubricants). Patients thought reassurance could be provided through an updatable FAQ section, sharing of patient experiences as well as through an interactive Q\&A 
section to engage with clinical staff. In instances where a patient did not feel reassured there should be clear signposting on where/who to contact next.

There are questions about the feasibility of this, the impact on staff and their workload and responsibility for monitoring, updating and responding to questions posed however, should that element be developed within the intervention, it would be explored further.

Patients requested a space within the platform to ask questions without worrying friends/family as well as a space to note questions for future appointments.

Patients reflected on how good it was to meet other women in the workshops with similar experiences and were keen to have a space to connect with peers. Suggested ideas included an events calendar or noticeboard of activities taking place for use to meet others or a space to organise their own activities enabling them to draw on social support from others with similar experiences. However, staff discussed (financial) barriers to attending activities so advocated for further options for connecting.

\section{Design Solution}

From the wish list, the initial design solution concentrated on four main areas: Advice and Support, Journal, Events and Connect.

Advice and Support: an information repository containing clearly defined subsections relating to areas of support required. Information would include things not necessarily portrayed in standard leaflets, tips and advice other women felt useful, as well as information directed at significant others. This would be tethered to current information practices to ensure currency, with consideration of cross-population of information from existing digital information.

Journal: somewhere to jot down thoughts, feelings and/or symptoms including; things to share with others (e.g. clinicians, family members); appointment notes and reminders; or somewhere to release niggling thoughts. The possibility of tracking symptoms within the app was considered as a separate area, represented by visual cues rather than text, potentially sent through to a nominated clinician if users wanted that.

Events: for users to create, share and 'book on' upcoming events in their area. This might include community groups, support groups or social gatherings, giving users the option of building local networks for further support. Once logged in, users could see events in their local area as well as create an event and 'invite' other users of the platform.

Connect/Profile: enables registered users to share demographic information in order to connect with other users at their own discretion (privacy settings were included in the terms and conditions of the intervention). With permission, other users of the platform would be visible with a username and location only so that a user can 'request' that person to connect with. Individuals can choose to accept the request, after which they see a more detailed profile, including contact details. Any contact would be made outside of the intervention through details shared.

Included within the platform was the function to submit a question. Generic answers would be provided by professionals and then posted on the platform FAQs for all users to see. Additionally, the platform would link out to a community forum, such as Health Unlocked, where communities can form around a particular health topic for further support and networking. It was decided an integrated forum within the platform would create excessive 
management issues which were not feasible, therefore a robust, existing forum was considered the best option.

\section{First Stage Evaluation}

Once the wireframes had been developed, a paper prototype was presented to a mixed staff, patient and researcher group. Key points from this session are indicated in Box 1.

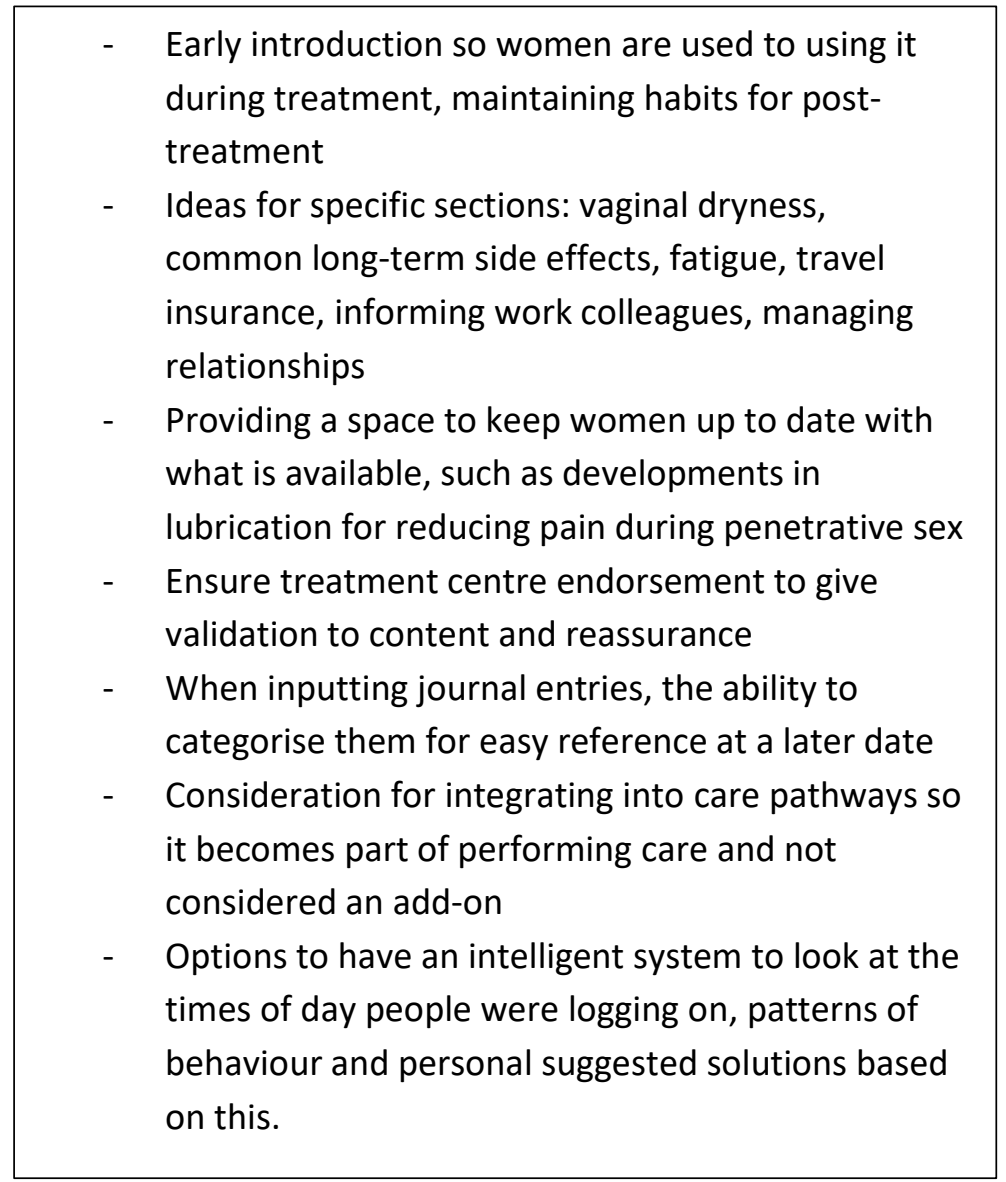

Box 1: Key Messages from Presentation of Paper Prototypes

Following the paper prototyping session, recommendations were made to the design build team who created an interactive prototype. This can be accessed at: https://xd.adobe.com/view/1484238d-5dc3-4c0e-7521-ce4ab0115249-62b5/?fullscreen

\section{Discussion}

The results of the project corroborated previous research that there is a gap in support for patients after radiotherapy treatment. Participants recognised a digital intervention would be an invaluable support tool post-treatment, in addition to the care they already receive, recognising that it would not replace existing face-to-face care. Whilst the project found other websites available for supporting patients with gynaecological cancer, these often focussed on one cancer site, for example cervical cancer, and women with less common diagnosis were uncatered for. Other websites also predominantly focussed on supporting women before and during treatment with little support for the long term, living with the physical and emotional effects of treatment. The intervention designed as part of this project was designed as a native app, rather than a website, so that it can be accessed easily 
and simply on a mobile device. This was based on discussions with women in the design sessions where women stated they would want to access it and all its functions on a mobile device without having to use a desktop or laptop. This was particularly relevant for women who often felt the need to search for information and support late at night. A key and unique element of the digital intervention was the journal function. This provided a place where women could note down thoughts, feelings, emotions, questions all in one place and tag them against a set of categories to make it easier to remember and categorise for their next hospital appointment.

The outcomes of the co-creation process go some way to meet the guiding principles for patient, public and practitioner partnerships within imaging and radiotherapy, as set out by the Society and College of Radiographers (26). Specifically, the methods for the design build expressly included patients and staff with all voices treated equally and regarded as key contributors to design.

The outcome intervention produced is grounded in an ethos of partnerships within care such as, providing patients with high-quality information, taking into consideration family members and carers. It was striking how beneficial staff members found listening to reallife, authentic patient experiences in a multidisciplinary context. There were immediate outcomes for patient care derived from these conversations that were unexpected consequences of the workshops.

\section{Conclusions}

The aim was to co-create an early prototype to support women who have had treatment for gynaecological cancer. Through workshops with multi-professional staff groups and women who have received treatment for gynaecological cancer, the effects of treatment and posttreatment support available were explored. Using a range of elicitation and discussion techniques it was found that support for women post-radiotherapy is lacking.

Collaborating with participants, a 'wish list' for the digital health intervention was developed and an early prototype was created. The prototype generated further discussion and informed interactive prototype development. Based on workshop findings, the project team believe that the co-created intervention offers the potential to provide life-long support for women living with and beyond cancer.

Although only five former patients and five staff members were included in the project, the methodology was robust in demonstrating the value patients and the public can bring to cocreation and ways to work in partnership in developing future care and support. The purposive approach to staff recruitment and convenience sampling of patients shaped the project and outcomes. However, this project was not aiming to generate generalizable findings. Rather, the aim was to conduct needs elicitation and develop an early prototype to demonstrate the opportunity space for further development.

Due to the time frame and funding for the project, further testing of the prototype has not taken place. Further funding will be sought to develop the prototype and to run implementation studies, exploring how the intervention works in practice and adjusting design and user experience along the way. In addition, the project team identified that indepth understanding of patient experiences of radiotherapy is lacking. This is the basis for an ongoing study (for details see [website]). 
In future stages, including funding applications and project design, patient, public and staff groups will be included. Co-creation is a rewarding and fulfilling activity and meets numerous aims beyond those of the project. It is recommended that mixed staff-patient groups are developed and adopted in more informal ways for the improvement of services. 


\section{Appendix A: Guides for Workshops}

\section{Example Prompt Questions for Initial Patient Workshops}

We would like to discuss well-being during and after your treatment.

The following factors are considered to affect well-being (30). Thinking about the period of time during and after your radiotherapy treatment, were any of the following affected

- Happy intimate relationship with a partner

- Network of close friends

- Enjoyable and fulfilling career

- Enough money

- Regular exercise

- Nutritional diet

- Sufficient sleep

- Spiritual or religious beliefs

- Fun hobbies and leisure pursuits

- Healthy self-esteem

- Optimistic outlook

- Realistic and achievable goals

- Sense of purpose and meaning

- A sense of belonging

- The ability to adapt to change

- Living in a fair and democratic society.

Were there any specific points you can identify when you felt your wellbeing was particularly low? For example, comparing during treatment, immediately afterwards or during the months after completion of treatment?

At those points, how were aspects of your wellbeing addressed? How informed or prepared did you feel about potential changes to wellbeing during or after treatment?

With relation to wellbeing, what have you learnt from your treatment experiences that you would like to feed into this project?

\section{Example Prompt Questions for Initial Staff Workshops}

What factors do you think affect wellbeing of patients during treatment?

How well do you think patients' wellbeing is supported?

What support for patients are you aware of?

Are there any specific periods (in the treatment and post treatment period) when you think wellbeing of women receiving treatment for gynaecological cancer aren't supported? Do you feel well equipped to support wellbeing of women receiving treatment for gynaecological cancer? 
EMPATHY MAP

\section{Tell us about your wellbeing...}

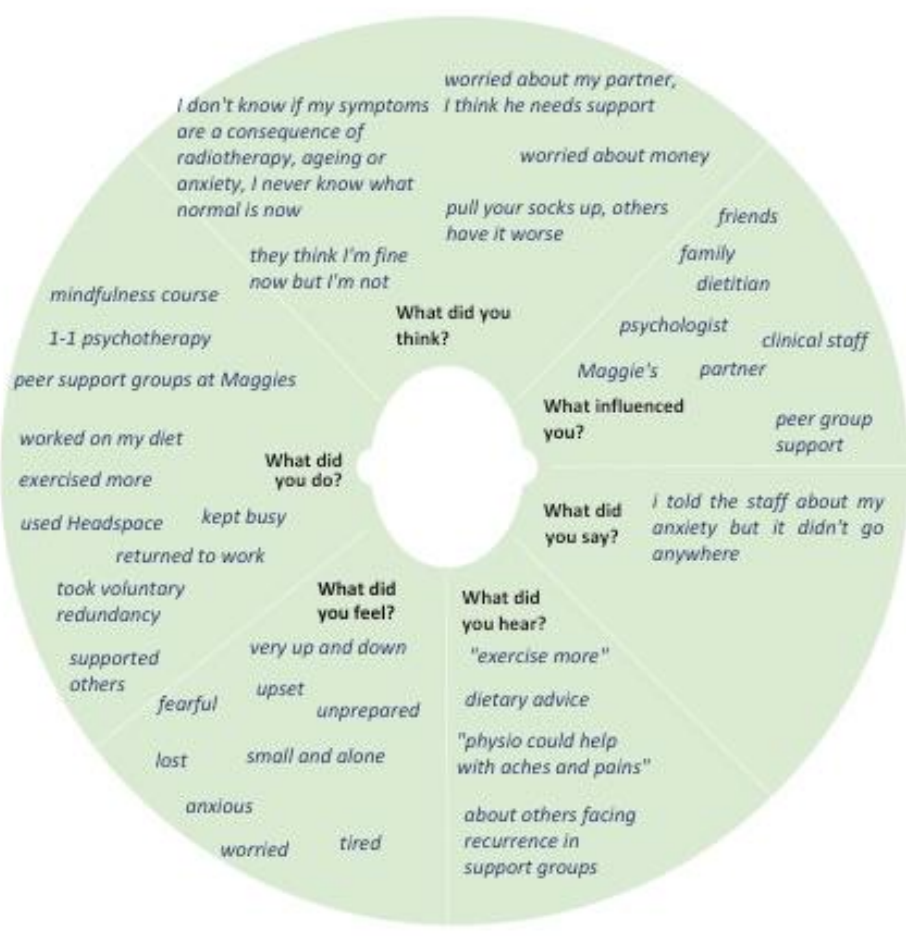

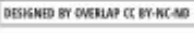

How prepared did you feel when your treament ended? - I wasn't prepored for the after effects.

- Once you've had your treotment, you ring the bell and you go, and fighting with you, looking ofter you, jollying you olong through treatment and then they soy "off you go"... well, where do /go? Whot do / do now? I went home and just muddled through. - There isn't realliy any preparation... if Maggie's wosn't there whot wouldi ido?

\section{How did you rebuild yourself?}

-Tlooked for anchors in my iffe, I tried to get a routine to help, I ushed back to work to try and feel some normanty, to keep myseff busy and keep my mind off things but thot just left me feeling

reactice the techniques from mindfulness but / hove to - mindfuiness; 1-1 psychotherapy; peer support groups; diet exercise; headspace \& meditation.

How have you adjusted to your new normal?

-I don't know what normal is anymore...
-It was a long time before I could enjoy things, if felt as though i lost

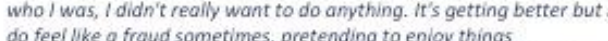

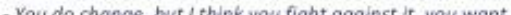

to wha you were, it's like you're a different person it's ilike i hove to do things differently now because ive had cancer.. it's the first thing that comes into your head

\section{What would have helped? \\ -If there was something / could use on the bad days when I'm strugging, san in the morkt, people do support there would be good And support to get back on the rood agoin and during the transitions from diagnosis to treatment to recovery...}

Overlap 


\section{References}

1. Henry DH, Viswanathan HN, Elkin EP, Traina S, Wade S, Cella D. Symptoms and treatment burden associated with cancer treatment: results from a cross-sectional national survey in the US. Supportive care in cancer. 2008;16(7):791-801.

2. Fang P, Tan KS, Grover S, McFadien MK, Troxel AB, Lin L. Psychosocial encounters correlates with higher patient-reported functional quality of life in gynecological cancer patients receiving radiotherapy. Radiation oncology. 2015;10(1):34.

3. Grigsby PW, Russell A, Bruner D, Eifel P, Koh W-J, Spanos W, et al. Late injury of cancer therapy on the female reproductive tract. International Journal of Radiation Oncology* Biology* Physics. 1995;31(5):1281-99.

4. Stone PC, Minton O. Cancer-related fatigue. European journal of cancer. 2008;44(8):1097-104.

5. Steineck G, Skokic V, Sjöberg F, Bull C, Alevronta E, Dunberger G, et al. Identifying radiation-induced survivorship syndromes affecting bowel health in a cohort of gynecological cancer survivors. PLoS One. 2017;12(2).

6. Barker CL, Routledge JA, Farnell DJ, Swindell R, Davidson SE. The impact of radiotherapy late effects on quality of life in gynaecological cancer patients. British journal of cancer. 2009;100(10):1558-65.

7. Curt GA, Breitbart W, Cella D, Groopman JE, Horning SJ, Itri LM, et al. Impact of cancer-related fatigue on the lives of patients: new findings from the Fatigue Coalition. The oncologist. 2000;5(5):353-60.

8. Bodurka DC, Sun CC. Sexual function after gynecologic cancer. Obstetrics and Gynecology Clinics. 2006;33(4):621-30.

9. Burns M, Costello J, RYAN-WOOLLEY B, Davidson S. Assessing the impact of late treatment effects in cervical cancer: an exploratory study of women's sexuality. European Journal of Cancer Care. 2007;16(4):364-72.

10. Conway JL, Gerber R, Han K, Jiang H, Xie J, Beiki-Ardakani A, et al. Patient-reported sexual adjustment after definitive chemoradiation and MR-guided brachytherapy for cervical cancer. Brachytherapy. 2019;18(2):133-40.

11. Pötter R, Georg P, Dimopoulos JC, Grimm M, Berger D, Nesvacil N, et al. Clinical outcome of protocol based image (MRI) guided adaptive brachytherapy combined with 3D conformal radiotherapy with or without chemotherapy in patients with locally advanced cervical cancer. Radiotherapy and Oncology. 2011;100:116-23.

12. Adams E, Boulton M, Horne A, Rose P, Durrant L, Collingwood M, et al. The effects of pelvic radiotherapy on cancer survivors: symptom profile, psychological morbidity and quality of life. Clinical Oncology. 2014;26(1):10-7.

13. Sandsund C., Pattison N., N. D, C. S. Finding a new normal: a grounded theory study of rehabilitation after treatment for upper gastrointestinal or gynaecological cancers - the patient's perspective. . European Journal of Cancer Care 2013;22:232-44.

14. Sandsund C, Towers R, Thomas K, Tigue R, Lalji A, Fernandes A, et al. Holistic needs assessment and care plans for women with gynaecological cancer: do they improve cancer- 
specific health-related quality of life? A randomised controlled trial using mixed methods. . BMJ Supportive \& Palliative Care. 2017;0:1-14.

15. Pfaendler KS, Wenzel L, Mechanic MB, Penner KR. Cervical cancer survivorship: longterm quality of life and social support. Clinical therapeutics. 2015;37(1):39-48.

16. Bakker RM, Mens JWM, de Groot HE, et al. A nurse-led sexual rehabilitation intervention after radiotherapy for gynecological cancer. Support Care Cancer 2017;25:729.

17. Vermeer W, Rinske M, Bakker M, Stiggelbout AM, Creutzberg CL, Kenter GG, et al. Psychosexual support for gynecological cancer survivors: professionals' current practices and need for assistance. Supportive Care in Cancer. 2015;23(3):831-9.

18. NHS. NHS Long Term Plan London: NHS; 2019 [Available from: https://www.longtermplan.nhs.uk/wp-content/uploads/2019/08/nhs-long-term-planversion-1.2.pdf.

19. NHS England. Achieving world-class cancer outcomes: a strategy for England 20152020. London, UK. 2015.

20. Sillence E, Briggs P, Harris PR, Fishwick L. How do patients evaluate and make use of online health information? Social science \& medicine. 2007;64(9):1853-62.

21. Wright KB, Bell SB. Health-related support groups on the Internet: Linking empirical findings to social support and computer-mediated communication theory. Journal of Health Psychology. 2003;8(1):39-54.

22. Lieberman MA, Wizlenberg A, Golant M, Di Minno M. The impact of group composition on Internet support groups: Homogeneous versus heterogeneous Parkinson's groups. . Group Dynamics: Theory, Research, and Practice. 2005;9(4):239.

23. The King's Fund. Digital Health Care: Our Position 2019 [Available from: https://www.kingsfund.org.uk/projects/positions/digital-health-care.

24. World Health Organization. Classification of Digital Health Interventions v1.0. Geneva: World Health Organization; 2018. Contract No.: WHO/RHR/18.06.

25. NHS. Five Year Forward View. 2014.

26. Society and College of Radiographers. Patient Public and Practitioner Partnerships within Imaging and Radiotherapy: Guiding Principles. 2017.

27. Abras C, Maloney-Krichmar D, Preece J. User-centered design. Bainbridge, W Encyclopedia of Human-Computer Interaction Thousand Oaks: Sage Publications. 2004;37(4):445-56.

28. (NIHR) NIfHR. National Standards for Public Involvement in Research. 2019.

29. Castro Llanos JW, Acuña ST, Juristo N, editors. Enriching requirements analysis with the Personas technique. CEUR Workshop Proceedings 2008.

30. Victoria State Government. Factors That Influence Well-Being 2014 [Available from: https://www.betterhealth.vic.gov.au/health/healthyliving/wellbeing\#lp-h-0 